Research Article

\title{
Curvature- and Torsion-Based Consensus for the Markovian Switching Multiagent System with Communication of Noise Disturbance and Time Delay
}

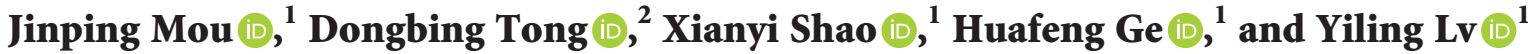 \\ ${ }^{1}$ School of the Electronics and Information Engineering, Taizhou University, Zhejiang 318000, China \\ ${ }^{2}$ College of Electronic and Electrical Engineering, Shanghai University of Engineering Science, Shanghai 201620, China
}

Correspondence should be addressed to Jinping Mou; mjptougaozhuanyong@163.com

Received 25 July 2020; Revised 1 March 2021; Accepted 5 March 2021; Published 25 March 2021

Academic Editor: Higinio Ramos

Copyright (C) 2021 Jinping Mou et al. This is an open access article distributed under the Creative Commons Attribution License, which permits unrestricted use, distribution, and reproduction in any medium, provided the original work is properly cited.

This paper investigates the consensus problem for the distributed multiagent system (MAS), where the trajectory of each agent is displayed by curvature and torsion, and the communication behaviors among agents are influenced by time delay and corrupted by noises. According to the Frenet-Serret formulas, a class of consensus protocols is designed for all agents, and a closed-loop system is obtained. Based on the Lyapunov method, several consensus criteria are derived, where the consensus criteria are characterized by curvature functions and torsion functions. Finally, one example shows the reliability of the proposed methods.

\section{Introduction}

In recent decades, the consensus problem of the multiagent system (MAS) has attracted the increasing attention of a large number of scholars, and the results have been widely used for tracking [1-5], formation control [6], distributed optimization monitoring $[7,8]$, and other purposes $[9,10]$.

The consensus of MAS requires that the state of each node tends to be a common value. Generally, the consensus problem is to design the distributed protocol for MAS [11], and under the protocol, the states of all agents converge to a common value by the local communication. However, for the abrupt change of topology and stochastic disturbance, the local communication will encounter link failures in some real applications. It is well known that the switching topology is widely described by Markovian chain.

Recently, many consensus results with Markovian switching have been investigated for MAS [12-23]. In [12], transition rates are considered to be not completely known; by the stochastic technique, sufficient conditions are expressed to solve the consensus problem for nonlinear MAS under the semi-Markovian switching topologies. In order to reduce the effect of communication noises, a time- varying gain in control protocol is utilized, and necessary and sufficient criteria are established to guarantee the mean square scaled consensus [13]. Considering the consensus control of grouped agents with linear or linearized nominal dynamics, a class of state-feedback controllers is proposed, and it shows that the controllers yield unbounded consensus region (see [14] and the references therein).

Group-couple consensus with the Markovian switching system is a duration-significant research field. In [18], by employing linear transformations, couple-group consensus is considered for a class of discrete-time stochastic multiagent systems, and the performance of the reduced-order systems is provided to get constructive approaches for the fixed and the Markovian switching topologies. Under a designed switching signal set, the group consensus problem of the second-order MAS is studied in [24], where the switching topology obeys the Markovian chain, and by utilizing a state transformation method, two consensus sufficient criteria are established. Based on a system transformation, the problem of group-couple is converted to a L2 control issue, and sufficient conditions are obtained for MAS, where the topology obeys a Markovian switching with unknown transition probabilities [17]. 
Event-triggered consensus problem with Markovian switching is another important issue on MAS, and it has attracted many scholars to study. In [25], in order to simplify information transmission, a distributed event-triggered mechanism is considered, and the proposed consensus protocol ensures MAS to achieve the consensus. By presenting an event-triggered control strategy, MAS achieves the fixed-time consensus [26-28].

Fault-tolerant tracking is another research field of MSA. In [29], an adaptive fault-tolerant tracking protocol is proposed, and it ensures each follower agent to track a leader agent. . By considering the directed link failures or recoveries, a fault-tolerant control strategy with Markovian switching topology is provided for a nonlinear MAS, and some sufficient conditions of stochastic consensus are obtained, where MAS is in the presence of communication noises and actuator faults [30].

Notice that most of the results mentioned above were obtained via the eigenvalue of matrix or the LMI method. In order to get the criteria of the consensus for MAS, some inherent relations will be neglected because of the inequalities to be utilized, such as curvature function and torsion function of each node when we consider the track problems. According to the theory of classic differential geometry, all tracks of agents are decided by their curvature and torsion entirely except for the difference of their locations. If all agents keep the same curvature function and torsion function, then they can move with the same track, and they can keep a certain distance among them. Recently, the consensus problem of MAS in the sense of curvature and torsion with fixed topology was studied in literature [31]. However, the results may not suitable to the switching system with stochastic disturbance. Motivated by this, in this paper, curvature- and torsion-based consensus problem with switching topology and noise disturbance is studied.

The remainder of this paper is organized as follows. Section 2 introduces some concepts. Section 3 proposes the consensus problem with Markovian switching. In Section 4, several consensus criteria are proposed for MAS. Section 5 provides a numerical example to show the reliability of the proposed methods. In Section 6, several conclusions are drawn.

Notations. The main notations which will be used in this paper are listed in the following. $Z^{+}$is the positive integer set, $R^{n}$ is the $n$-dimensional Euclidean space, $C^{3}$ is the 3 -times continuously differentiable, $I_{n}$ is the $n$ times $n$ unit matrix, and $\mathbf{0}$ is the zero vector.

\section{Preliminaries}

Let $\mathscr{V}=\left\{v_{1}, v_{2}, \ldots, v_{i}, \ldots, v_{n}\right\}$ be the agent set, where $i \in Z^{+}$, $1 \leq i \leq n$. Let $\mathscr{A}=\left(a_{i j}\right)_{n \times n}$ be a weighted symmetric adjacent matrix of all agents, where $a_{i j}$ is associated with an edge which is denoted by $(i, j) . a_{i j}$ takes value in 1 or 0 , and $a_{i j}=a_{j i} . a_{i j}=1$ refers to the fact that there exists information flow between nodes $v_{j}$ and $v_{i}$, or else $a_{i j}=0$. Let $\mathscr{E}=\left\{(i, j) \mid a_{i j}=1, i \neq j\right\}$ be the edge set, where $(i, j)$ is the edge between $v_{i}$ and $v_{j}$. The neighbor set of $v_{i}$ is denoted by $N_{i}$, where $N_{i}=\left\{v_{j} \mid\left(v_{i}, v_{j}\right) \in \mathscr{E}\right\}$.

Based on $\mathscr{V}, \mathscr{A}$, and $\mathscr{E}$, the topology of the multiagent system (MAS) can be presented by a weighted graph $\mathscr{G}=(\mathscr{V}, \mathscr{E}, \mathscr{A})$.

Let $\mathscr{G}\left(\varrho_{l}\right)=\left(\mathscr{V}, \mathscr{E}\left(\varrho_{l}\right), \mathscr{A}\left(\varrho_{l}\right)\right)$ be the interaction topology of dynamic agents, where

$$
\varrho(t)=\varrho_{l}, \quad t \in[t, t+a), a>0,
$$

and $\mathscr{G}\left(\varrho_{l}\right)$ refers to the time-varying topology which is driven by a random press $\varrho(t)$, and $\varrho_{l} \in \mathscr{T}$, where $\mathscr{T}=\left\{\varrho_{1}, \varrho_{2}, \ldots, \varrho_{i}, \ldots \varrho_{m}\right\}$. The infinitesimal generator of the Markov chain $\{\varrho(t), t \geq 0\}$ is given by

$$
P\left\{\varrho(t+a)=\varrho_{j} \mid \varrho_{i}\right\}= \begin{cases}\lambda_{i j} h+o(h), & \text { if } i \neq j, \\ 1+\lambda_{i i} h+o(h), & \text { otherwise, }\end{cases}
$$

where $\lambda_{i j}$ is the transition rate from state $\varrho_{i}$ to $\varrho_{j}$; if $\varrho_{i} \neq \varrho_{j}$, then $\lambda_{i j} \geq 0, \sum_{i \neq j} \lambda_{i j}$, and $\lim _{h \longrightarrow 0}(o(h) / h)=0 . \forall v_{i} \in \mathscr{V}$, let

$$
r_{i}(t)=\left\{x_{i}(t), y_{i}(t), z_{i}(t)\right\}^{T} \in R^{3}
$$

be the state of agent $v_{i}$, where $t \in R$, and $x_{i}(t), y_{i}(t), z_{i}(t)$ are the $C^{3}$ functions; hence, the state of MAS is shown by

$$
r(t)=\left\{r_{1}(t)^{T}, r_{2}(t)^{T}, \ldots, r_{i}(t)^{T}, \ldots, r_{n}(t)^{T}\right\}^{T} .
$$

Denote

$$
\begin{aligned}
& \alpha_{i}(t)=\frac{r^{\prime}(t)}{\left\|r^{\prime}(t)\right\|}, \\
& \gamma_{i}(t)=\frac{r^{\prime}(t) \times r^{\prime \prime}(t)}{\left\|r^{\prime}(t) \times r^{\prime \prime}(t)\right\|}, \\
& \beta_{i}(t)=\gamma_{i}(t) \times \alpha_{i}(t),
\end{aligned}
$$

where $\alpha_{i}(t)$ is the tangent unit vector, $\beta_{i}(t)$ is the normal unit vector, and $\gamma_{i}(t)$ is the binormal unit vector, and they satisfy the following Frenet-Serret formulas:

$$
\left\{\begin{array}{l}
\alpha_{i}^{\prime}(t)=\kappa_{i}(t) \beta_{i}(t) \\
\beta_{i}^{\prime}(t)=-\kappa_{i}(t) \alpha_{i}(t)+\tau_{i}(t) \gamma_{i}(t) \\
\gamma_{i}^{\prime}(t)=-\tau_{i}(t) \beta_{i}(t)
\end{array}\right.
$$

where $\kappa_{i}(t)$ and $\tau_{i}(t)$ are the curvature function and torsion function of $v_{i}$, respectively.

As is known to all, the trajectory of $v_{i}$ is decided entirely by the curvature function and the torsion function. Therefore, $\forall v_{i}$ and $v_{j}$, if

$$
\begin{gathered}
\kappa_{i}(t)=\kappa_{j}(t), \\
\tau_{i}(t)=\tau_{j}(t),
\end{gathered}
$$

then $v_{i}$ and $v_{j}$ have the same trajectory except for the difference of their locations.

For convenience, we denote $\widehat{\tau}=\max \left\{\tau_{i}(t) \mid 1 \leq i \leq n\right\}$, $\widehat{\kappa}=\max \left\{\kappa_{i}(t) \mid 1 \leq i \leq n\right\}, \check{\tau}=\min \left\{\tau_{i}(t) \mid 1 \leq i \leq n\right\}, \check{\kappa}=\min \left\{\kappa_{i}\right.$ $(t) \mid 1 \leq i \leq n\}$. 
Assumption 1. We assume that

$$
\begin{array}{ll}
\kappa_{i}^{\prime}(t)=0, & t \in[t, t+a), \\
\tau_{i}^{\prime}(t)=0, & t \in[t, t+a) .
\end{array}
$$

Consequently, based on the curvature function and torsion function, a consensus definition is proposed in the following.

Definition 1. $\forall v_{i}, v_{j} \in \mathscr{V}$, if

$$
\begin{aligned}
& \lim _{t \longrightarrow \infty} E\left(\left\|\kappa_{i}(t)-\kappa_{j}(t)\right\|\right)=0, \\
& \lim _{t \longrightarrow \infty} E\left(\left\|\tau_{i}(t)-\tau_{j}(t)\right\|\right)=0,
\end{aligned}
$$

then MAS is the consensus in a broad sense of curvature and torsion (BCT consensus).

Remark 1. In literature [31], CT consensus in the sense of curvature and torsion refers to the fact that

$$
\begin{aligned}
& \lim _{t \longrightarrow \infty}\left(\kappa_{i}(t)-\kappa_{j}(t)\right)=0, \\
& \lim _{t \longrightarrow \infty}\left(\tau_{i}(t)-\tau_{j}(t)\right)=0,
\end{aligned}
$$

and hence $C T$ consensus is the special case of $B C T$ consensus.

Remark 2. In the traditional literatures, e.g., see $[8,32]$, the consensus refers to the fact that $\forall v_{i}, v_{j} \in \mathscr{V}$,

$$
\lim _{t \rightarrow \infty}\left[r_{i}(t)-r_{j}(t)\right]=\mathbf{0},
$$

and it follows that

$$
\begin{aligned}
& \lim _{t \longrightarrow \infty}\left[\kappa_{i}(t)-\kappa_{j}(t)\right]=0, \\
& \lim _{t \longrightarrow \infty}\left[\tau_{i}(t)-\tau_{j}(t)\right]=0,
\end{aligned}
$$

and the state error between every two agents converges to zero vector. However, in some applications, we need all agents to keep nonzero distances while their trajectories possess the same shape.

According to the Definition 1, every two agents can keep the nonzero distance. Meanwhile, all trajectories of agents converge to an interest curve.

Remark 3. In Definition 1, if

$$
\begin{aligned}
& \lim _{t \longrightarrow \infty} E\left(\left\|\alpha_{i}(t)-\alpha_{j}(t)\right\|\right)=0, \\
& \lim _{t \longrightarrow \infty} E\left(\left\|\beta_{i}(t)-\beta_{j}(t)\right\|\right)=0, \\
& \lim _{t \longrightarrow \infty} E\left(\left\|\gamma_{i}(t)-\gamma_{j}(t)\right\|\right)=0,
\end{aligned}
$$

based on equation (6), it follows that

$$
\beta_{i}(t) \cdot \alpha_{i}^{\prime}(t)=\kappa_{i}(t),
$$

and it holds that

$$
\lim _{t \longrightarrow \infty} E\left(\left\|\kappa_{i}(t)-\kappa_{j}(t)\right\|\right)=0 .
$$

Similarly, it gets that

$$
\lim _{t \longrightarrow \infty} E\left(\left\|\tau_{i}(t)-\tau_{j}(t)\right\|\right)=0 .
$$

\section{Problem Statement}

In this section, the protocols are provided for tangent vector, normal vector, and binormal vectors firstly, and then the dynamics of tangent vectors, normal vectors, and binormal vectors are described for MAS.

$\forall v_{i} \in \mathscr{V}$, the dynamics of $\alpha_{i}(t), \beta_{i}(t), \gamma_{i}(t)$ are described by

$$
\left\{\begin{array}{l}
\alpha_{i}^{\prime}(t)=\kappa_{i}(t) \beta_{i}(t)+u_{i}^{1}(t), \\
\beta_{i}^{\prime}(t)=-\kappa_{i}(t) \alpha_{i}(t)+\tau_{i}(t) \gamma_{i}(t)+u_{i}^{2}(t), \\
\gamma_{i}^{\prime}(t)=-\tau_{i}(t) \beta_{i}(t)+u_{i}^{3}(t)
\end{array}\right.
$$

where $\forall t \in[s, s+a), \kappa_{i}^{\prime}(t)=0, \tau_{i}^{\prime}(t)=0, u_{i}^{1}(t), u_{i}^{2}(t)$, and $u_{i}^{3}(t)$ are the protocols of agent $v_{i}$, and they are defined by

$$
\left\{\begin{array}{l}
u_{i}^{1}(t)=\kappa_{i}(t-a) \sum_{j \in N_{i}} a_{i j}\left[\alpha_{j}(t-a)-\alpha_{i}(t-a)+\sigma(t) w_{i j}\right]-\frac{1}{d_{i}} \sum_{j \in N_{i}} a_{i j}\left[\kappa_{i}(t) \beta_{j}(t)\right], \\
u_{i}^{2}(t)=\left[\tau_{i}(t-a)-\kappa_{i}(t-a)\right] \sum_{j \in N_{i}} a_{i j}\left[\beta_{j}(t-a)-\beta_{i}(t-a)+\sigma(t) w_{i j}\right]+\frac{1}{d_{i}} \sum_{j \in N_{i}} a_{i j}\left[\kappa_{i}(t) \alpha_{j}(t)-\tau_{i}(t) \gamma_{j}(t)\right], \\
u_{i}^{3}(t)=\tau_{i}(t-a) \sum_{j \in N_{i}} a_{i j}\left[\gamma_{j}(t-a)-\gamma_{i}(t-a)+\sigma(t) w_{i j}\right]+\frac{1}{d_{i}} \sum_{j \in N_{i}} a_{i j}\left[\tau_{i}(t) \beta_{j}(t)\right],
\end{array}\right.
$$


where $w_{i j}$ is the white noise, $\sigma(t) \geq 0$, and random press $\varrho(t)$ and $w_{i j}$ are independent of each other.

From (17) and (18), we obtain that

$$
\begin{aligned}
{\left[\begin{array}{l}
\alpha^{\prime}(t) \\
\beta^{\prime}(t) \\
\gamma^{\prime}(t)
\end{array}\right]=} & {\left[\begin{array}{ccc}
0 & \Pi_{12} & 0 \\
\Pi_{21} & 0 & \Pi_{23} \\
0 & \Pi_{32} & 0
\end{array}\right]\left[\begin{array}{l}
\alpha(t) \\
\beta(t) \\
\gamma(t)
\end{array}\right] } \\
& -\left[\begin{array}{ccc}
K\left(\varrho_{l}\right) L\left(\varrho_{l}\right) & 0 & 0 \\
0 & -\left[T\left(\varrho_{l}\right)-K\left(\varrho_{l}\right)\right] L\left(\varrho_{l}\right) & 0 \\
0 & 0 & T\left(\varrho_{l}\right) L\left(\varrho_{l}\right)
\end{array}\right] \\
& \cdot\left[\begin{array}{c}
\alpha(t-a) \\
\beta(t-a) \\
\gamma(t-a)
\end{array}\right] \\
& +\left[\begin{array}{ccc}
\Lambda_{11} & 0 & 0 \\
0 & \Lambda_{22} & 0 \\
0 & 0 & \Lambda_{33}
\end{array}\right] \sigma(t) C W,
\end{aligned}
$$

where

$$
\begin{aligned}
\alpha & =\left(\alpha_{1}^{T}(t), \alpha_{2}^{T}(t), \ldots, \alpha_{n}^{T}(t)\right)^{T}, \\
\beta & =\left(\beta_{1}^{T}(t), \beta_{2}^{T}(t), \ldots, \beta_{n}^{T}(t)\right)^{T}, \\
\gamma & =\left(\gamma_{1}^{T}(t), \gamma_{2}^{T}(t), \ldots, \gamma_{n}^{T}(t)\right)^{T}, \\
W_{i}^{T} & =\left\{w_{i 0}, w_{i 1}, \ldots, w_{i n}\right\}^{T}, \\
W & =\left\{W_{1}^{T}, \ldots, W_{n}^{T}\right\}^{T} .
\end{aligned}
$$

Denote $X(t)=\left(\alpha^{T}(t), \beta^{T}(t), \gamma^{T}(t)\right)^{T}$, and we have

$X^{\prime}(t)=\Pi(t) X(t)-\Sigma(t) X(t-a)+\Sigma(t) \sigma(t) C W$,

where

$$
\begin{aligned}
\Pi & =\left[\begin{array}{ccc}
0 & \Pi_{12} & 0 \\
\Pi_{21} & 0 & \Pi_{23} \\
0 & \Pi_{32} & 0
\end{array}\right], \\
\Sigma(t) & =\left[\begin{array}{lll}
\Sigma_{1} & 0 & 0 \\
0 & \Sigma_{2} & 0 \\
0 & 0 & \Sigma_{3}
\end{array}\right], \\
D\left(\varrho_{l}\right) & =\operatorname{diag}\left\{\frac{1}{d_{1}}, \frac{1}{d_{2}}, \ldots, \frac{1}{d_{n}}\right\}, \\
\Pi_{12} & =D K\left(\varrho_{l}\right) L\left(\varrho_{l}\right), \\
\Pi_{21} & =-D\left(\varrho_{l}\right) K\left(\varrho_{l}\right) L\left(\varrho_{l}\right), \\
\Pi_{23} & =D\left(\varrho_{l}\right) T\left(\varrho_{l}\right) L\left(\varrho_{l}\right), \\
\Pi_{32} & =-D\left(\varrho_{l}\right) T\left(\varrho_{l}\right) L\left(\varrho_{l}\right), \\
\Sigma_{1} & =K\left(\varrho_{l}\right) L\left(\varrho_{l}\right), \\
\Sigma_{2} & =-\left[K\left(\varrho_{l}\right)-T\left(\varrho_{l}\right)\right] L\left(\varrho_{l}\right), \\
\Sigma_{3} & =T L\left(\varrho_{l}\right), \\
K\left(\varrho_{l}\right) & =\left\{\kappa_{1}(t), \kappa_{2}(t), \ldots, \kappa_{n}(t)\right\}, \\
T\left(\varrho_{l}\right) & =\left\{\tau_{1}(t), \tau_{2}(t), \ldots, \tau_{n}(t)\right\},
\end{aligned}
$$

where $C$ is a $n^{2} \times n$ matrix, and

$$
C=\left[\begin{array}{cccccccccc}
0 & a_{12} & \cdots & a_{1 n} & 0 & 0 & 0 & 0 & \cdots & 0 \\
0 & \cdots & 0 & a_{21} & 0 & \cdots & a_{2 n} & 0 & \cdots & 0 \\
\vdots & \vdots & \vdots & \vdots & \vdots & \vdots & \vdots & \vdots & \cdots & \vdots \\
0 & 0 & 0 & 0 & \cdots & 0 & 0 & a_{n 1} & \cdots & 0
\end{array}\right] .
$$

In the following, the BCT consensus of system (21) will be studied.

\section{The Main Results}

In this section, the consensus problem is studied for system (21). Firstly, the state error of system (21) is discussed. Let $x_{0}(t)=\left(\alpha_{0}(t), \beta_{0}(t), \gamma_{0}(t)\right)$ be the aim state of the MAS, where

$$
\begin{aligned}
& \alpha_{0}(t)=\frac{1}{n} \sum_{i=1}^{n} \alpha_{i}, \\
& \beta_{0}(t)=\frac{1}{n} \sum_{i=1}^{n} \beta_{i}, \\
& \gamma_{0}(t)=\frac{1}{n} \sum_{i=1}^{n} \gamma_{i} .
\end{aligned}
$$

Denote $\quad e(t)=X(t)-x_{0}(t) \otimes I_{n+1}=\left(\delta_{1}(t)^{T}, \delta_{2}(t)^{T}\right.$, $\left.\delta_{3}(t)^{T}\right)^{T}$; then, it follows that

$$
\begin{aligned}
& \delta_{1}(t)=\alpha(t)-\alpha_{0}(t), \\
& \delta_{2}(t)=\beta(t)-\beta_{0}(t), \\
& \delta_{3}(t)=\gamma(t)-\gamma_{0}(t),
\end{aligned}
$$

and it holds that

$$
e(t)=X(t)-x_{0}(t) \otimes I_{n+1}=(I-P) X(t),
$$

where

$$
\begin{gathered}
P=\left[\begin{array}{ccc}
P_{11} & 0 & 0 \\
0 & P_{22} & 0 \\
0 & 0 & P_{33}
\end{array}\right], \\
P_{11}=P_{22}=P_{33}=\frac{1}{n} \mathbf{1}_{n \times n} .
\end{gathered}
$$

It follows that

$$
\begin{aligned}
e^{\prime}(t)= & (I-P) X^{\prime}(t) \\
= & (I-P) \Pi X(t)-(I-P) \Sigma(t) X(t-a) \\
& +(I-P) \Sigma(t) \sigma(t) C W .
\end{aligned}
$$

Notice that $\left(\mathbf{1}_{n \times n} / n\right) L\left(\varrho_{\iota}\right)=0$, and 


$$
\begin{aligned}
\sum_{j \in N_{i}}\left[\alpha_{j}(t)-\alpha_{i}(t)\right] & =\sum_{j \in N_{i}}\left[\alpha_{j}(t)-\alpha_{0}(t)-\alpha_{i}(t)+\alpha_{0}(t)\right] \\
& =\sum_{j \in N_{i}}\left[e_{j}(t)-e_{i}(t)\right] .
\end{aligned}
$$

Hence, $L\left(\varrho_{l}\right) X(t)=L\left(\varrho_{\iota}\right) e(t)$, and system (28) can be shown by

$$
e^{\prime}(t)=(I-P) \Pi(t) e(t)+(I-P) \Sigma(t) e(t-a)+(I-P) \Sigma(t) C W,
$$

$$
d e(t)=f\left(\varrho_{l}, e(t), e(t-a)\right) \mathrm{d} t+g\left(\varrho_{l}, e(t), e(t-a)\right) d B(t),
$$

where

$$
\begin{aligned}
f\left(\varrho_{1}, e(t), e(t-a)\right)= & (I-P) \Pi(t) e(t) \\
& -(I-P) \Sigma(t)(t) e(t-a), \\
g\left(\varrho_{l}, e(t), e(t-a)\right)= & (I-P) \Sigma(t) \sigma(t) C .
\end{aligned}
$$

Lemma 1 (see [33]). Let $a^{\prime}(t) \leq \bar{a}(t>0)$ hold. Assume that there is a function $V \in C^{2,1}\left(R^{n} \times R_{+} ; R_{+}\right)$and positive constants $p, c_{1}, c_{2}, c_{3}, c_{4}$ such that $c_{3}>c_{4} /(1-\bar{a})$,

$$
\begin{aligned}
c_{1}|e(t)|^{p} & \leq V\left(e(t), t, \varrho_{\iota}\right) \leq c_{2}|e(t)|^{p}, \\
L V & \left.\leq(e(t), t,\rangle_{\iota}\right) \leq-\lambda_{1}\|e(t)\|^{p}+\lambda_{2}\|e(t-a)\|^{p},
\end{aligned}
$$

for all $e(t), e(t-a) \in R^{n}, t \geq 0$, and $\rho_{l} \in \mathscr{T}$. Then, equation (31) is asymptotically stable in pth moment.

Lemma 2 (see [33]). Let $a^{\prime}(t) \leq \bar{a}(t>0)$ hold and $p \geq 2$. Assume that for each $\iota \in \mathscr{T}$, there is a pair of real numbers $\varsigma_{\rho_{1}}$ and $\sigma_{\rho_{t}}$ such that

$$
\begin{aligned}
& e(t)^{T} f(e(t), e(t-a(t)))+\frac{p-1}{2}\|g(e(t), e(t-a(t)))\|^{2} \\
& \leq \varsigma_{\rho_{t}}\|e(t)\|^{2}+\sigma_{\rho_{t}}\|e(t-a)\|^{2},
\end{aligned}
$$

for all $(e(t), e(t-a), t) \in R^{n} \times R^{n} \times R_{+}$. Assume that

$$
\mathscr{B}:=-\operatorname{diag}\left\{p \varsigma_{\rho_{1}}, \ldots, p \varsigma_{\rho_{m}}\right\}-\Gamma
$$

is a nonsingular M-matrix, so $\left(q_{1}, \ldots, q_{m}\right)^{T}:=\mathscr{B}^{-1} \overrightarrow{1} \gg 0$. If

$$
p q_{\rho_{t}} \sigma_{\rho_{t}}<1-\bar{a}, \varsigma_{\rho_{t}} \in \mathscr{T},
$$

then equation (31) is a asymptotically stable in pth moment.

Then, we discuss the stability of system (31). Let the Lyapunov candidate function be presented as

$$
V(t)=\frac{1}{2} e(t)^{T} e(t)
$$

and it follows that

$$
\begin{aligned}
& e(t)^{T}[(I-P) \Pi(t) e(t)]=\left[\delta_{1}(t)^{T}, \delta_{2}(t)^{T}, \delta_{3}(t)^{T}\right](I-P) \Pi\left[\delta_{1}(t)^{T}, \delta_{2}(t)^{T}, \delta_{3}(t)^{T}\right]^{T} \\
& =-\delta_{2}(t)^{T}\left(I-P_{22}\right) \Pi_{12} \delta_{1}(t)+\delta_{1}(t)^{T}\left(I-P_{11}\right) \Pi_{12} \delta_{2}(t) \\
& -\delta_{3}(t)^{T}\left(I-P_{33}\right) \Pi_{23} \delta_{2}(t)+\delta_{2}(t)^{T}\left(I-P_{22}\right) \Pi_{23} \delta_{3}(t) \\
& =0 \text {, } \\
& e(t)^{T}\left[(I-P) \sum(t) e(t-a)\right]=\left[\delta_{1}(t)^{T}, \delta_{2}(t)^{T}, \delta_{3}(t)^{T}\right](I-P) \sum(t)\left[\delta_{1}(t-a)^{T}, \delta_{2}(t-a)^{T}, \delta_{3}(t-a)^{T}\right]^{T} \\
& =\sum_{i=1}^{3} \delta_{i}(t)^{T}\left(I-P_{i i}\right) \Sigma_{i}(t) \delta_{i}(t-a)=\sum_{i=1}^{3} \frac{1}{2}\left[\delta_{i}(t)^{T}\left(I-P_{i i}\right) \Sigma_{i}(t) \delta_{i}(t)+\delta_{i}(t-a)^{T}\left(I-P_{i i}\right) \Sigma_{i}(t) \delta_{i}(t-a)\right. \\
& \left.-\left(\delta_{i}(t)-\delta_{i}(t-a)\right)^{T}\left(I-P_{i i}\right) \Sigma_{i}(t)\left(\delta_{i}(t)-\delta_{i}(t-a)\right)\right] .
\end{aligned}
$$

To estimate the value of $e(t)^{T}[(I-P) \Sigma(t) e(t-a)]$, we need the following conditions.and we also need the following assumptions.

Assumption 2. In this paper, we assume that

$$
\widehat{\kappa} \geq \check{\tau} \geq 0,
$$

Assumption 3. Let $\tilde{\delta_{1}}, \quad \tilde{\delta_{2}}, \quad \tilde{\delta_{3}}$ satisfy the following inequalities:

$$
\begin{aligned}
& \left\|\tilde{\delta_{1}}\right\| \leq \sqrt{k_{1}}\left\|\widehat{\delta_{1}}\right\|, \\
& \left\|\tilde{\delta_{2}}\right\| \geq \sqrt{k_{2}}\left\|\delta_{2}\right\|, \\
& \left\|\tilde{\delta_{3}}\right\| \leq \sqrt{k_{3}}\left\|\widehat{\delta_{3}}\right\|,
\end{aligned}
$$


where $\widetilde{\delta}_{i}=\delta_{i}(t)-\delta_{i}(t-a)$.

Under Assumption 3, it holds that

$$
\begin{aligned}
\sum_{i=1}^{3} \delta_{i}(t)^{T}\left(I-P_{i i}\right) \Sigma_{i}(t) \delta_{i}(t) \leq \sum_{i=1}^{3}\left\|\left(I-P_{i i}\right) \Sigma_{i}(t)\right\|\left\|\delta_{i}(t)^{T} \delta_{i}(t)\right\| \\
e(t)^{T}[(I-P) \Sigma(t) e(t-a)] \\
=\left[\delta_{1}(t)^{T}, \delta_{2}(t)^{T}, \delta_{3}(t)^{T}\right](I-P) \Sigma(t)\left[\delta_{1}(t-a)^{T}, \delta_{2}(t-a)^{T}, \delta_{3}(t-a)^{T}\right]^{T} \\
=\sum_{i=1}^{3} \delta_{i}(t)^{T}\left(I-P_{i i}\right) \Sigma_{i}(t) \delta_{i}(t-a) \\
=\sum_{i=1}^{3} \frac{1}{2}\left[\delta_{i}(t)^{T}\left(I-P_{i i}\right) \Sigma_{i}(t) \delta_{i}(t)+\delta_{i}(t-a)^{T}\left(I-P_{i i}\right) \Sigma_{i}(t) \delta_{i}(t-a)\right. \\
\left.\quad-\left(\delta_{i}(t)-\delta_{i}(t-a)\right)^{T}\left(I-P_{i i}\right) \Sigma_{i}(t)\left(\delta_{i}(t)-\delta_{i}(t-a)\right)\right] .
\end{aligned}
$$

Notice that $\lambda\left(P_{i i} K L\right)=0$; then, it follows that

$$
\lambda\left[\left(I-P_{i i}\right) K L\right]=\lambda(K L),
$$

and we have

$$
\lambda_{2} \check{\kappa} \leq \lambda(K L) \leq \lambda_{M} \widehat{\kappa},
$$

$$
\begin{aligned}
&-\delta_{1}(t)^{T}\left(I-P_{1}\right) \Sigma_{1} \delta_{1}(t)=-\delta_{1}(t)^{T} K L \delta_{1}(t) \\
& \leq-\check{\kappa} \lambda_{2} \delta_{1}(t)^{T} \delta_{1}(t), \\
& \delta_{2}(t)^{T}\left(I-P_{2}\right) \Sigma_{2} \delta_{2}(t)=\delta_{2}(t)^{T} K L \delta_{2}(t)-\delta_{2}(t)^{T} T L \delta_{2}(t) \\
& \leq {\left[\widehat{\kappa} \lambda_{M}-\check{\tau} \lambda_{2}\right] \delta_{2}(t)^{T} \delta_{2}(t), } \\
&-\delta_{3}(t)^{T}\left(I-P_{3}\right) \Sigma_{3} \delta_{3}(t)=-\delta_{3}(t)^{T} K L \delta_{3}(t) \\
& \leq-\check{\tau} \lambda_{2} \delta_{3}(t)^{T} \delta_{3}(t) .
\end{aligned}
$$

Similarly, it follows that

$$
\begin{aligned}
& -\delta_{1}(t-a)^{T}\left(I-P_{1}\right) \Sigma_{1} \delta_{1}(t-a) \leq-\check{\kappa} \lambda_{2} \delta_{1}(t-a)^{T} \delta_{1}(t-a), \\
& \delta_{2}(t-a)^{T}\left(I-P_{2}\right) \Sigma_{2} \delta_{2}(t-a) \leq \widehat{\kappa} \lambda_{M} \delta_{2}(t-a)^{T} \delta_{2}(t-a)-\check{\tau} \lambda_{2} \delta_{2}(t-a)^{T} \delta_{2}(t-a) \\
& =\left(\widehat{\kappa} \lambda_{M} t-n \check{\tau} q \lambda_{2}\right) \delta_{2}(t-a)^{T} \delta_{2}(t-a), \\
& -\delta_{3}(t-a)^{T}\left(I-P_{3}\right) \Sigma_{3} \delta_{3}(t-a) \leq-\check{\tau} \lambda_{2} \delta_{3}(t-a)^{T} \delta_{3}(t-a) .
\end{aligned}
$$

Assumption 4. We assume that

$$
h_{i}(t) \delta_{i}^{T}(t) \delta_{i}(t) \leq \widetilde{\delta}_{i}^{T}(t) \widetilde{\delta}_{i}(t) \leq g_{i}(t) \delta_{i}^{T}(t) \delta_{i}(t)
$$

where $\widetilde{\delta}_{i}(t)=\delta_{i}(t)-\delta_{i}(t-a), h_{i}(t) \geq 0$ and $g_{i}(t) \geq 0$.

In addition, $\widetilde{\delta}_{i}(t),(i=1,2,3)$ satisfy the following inequalities: 


$$
\begin{aligned}
& \widetilde{\delta}_{1}(t)^{T}\left(I-P_{1}\right) \Sigma_{1} \widetilde{\delta}_{1}(t)=\widetilde{\delta}_{1}(t)^{T} K L \widetilde{\delta}_{1}(t) \\
& \leq \widehat{\kappa} \lambda_{M} \widetilde{\delta}_{1}(t)^{T} \widetilde{\delta}_{1}(t) \\
& \leq g_{i}(t) \widehat{\kappa} \lambda_{M} \delta_{1}(t)^{T} \delta_{1}(t) \\
& \widetilde{\delta}_{2}(t)^{T}\left(I-P_{2}\right) \Sigma_{2} \widetilde{\delta}_{2}(t)=-\widetilde{\delta}_{2}(t)^{T} K L \widetilde{\delta}_{2}(t)+\widetilde{\delta}_{2}(t)^{T} T L \widetilde{\delta}_{2}(t), \\
& \leq-h_{2}(t) \check{\kappa} \lambda_{2} \delta_{2}(t)^{T} \delta_{2}(t)+g_{2}(t) \widehat{\tau} \lambda_{2} \delta_{2}(t)^{T} \delta_{2}(t) \\
& =\left(-\check{\kappa} \lambda_{2} h_{2}(t)+\widehat{\tau} \lambda_{M} g_{2}(t)\right) \delta_{2}(t)^{T} \delta_{2}(t), \\
& \widetilde{\delta}_{3}(t)^{T}\left(I-P_{3}\right) \Sigma_{3} \widetilde{\delta}_{3}(t)=\widetilde{\delta}_{3}(t)^{T} K L \widetilde{\delta}_{3}(t) \\
& \leq \widehat{\tau} \lambda_{M} g_{3}(t) \delta_{3}(t)^{T} \delta_{3}(t) .
\end{aligned}
$$

For convenience, denote

$$
\begin{aligned}
& f_{1}\left(\delta_{1}\right)=\left[-\check{\kappa} \lambda_{2}+g_{i}(t) \widehat{\kappa} \lambda_{M}\right] \delta_{1}(t)^{T} \delta_{1}(t), \\
& f_{2}\left(\delta_{1}\right)=\left[\widehat{\kappa} \lambda_{M}-h_{2}(t) \check{\kappa} \lambda_{2}-\check{\tau} \lambda_{2}+\widehat{\tau} \lambda_{M} g_{2}(t)\right] \delta_{2}(t)^{T} \delta_{2}(t), \\
& f_{3}\left(\delta_{1}\right)=\left[-\check{\tau} \lambda_{2}+\widehat{\tau} \lambda_{M} g_{3}(t)\right] \delta_{3}(t)^{T} \delta_{3}(t) .
\end{aligned}
$$

On the other hand, it holds that

$$
\begin{aligned}
& \operatorname{trace}\left[((I-P) \Sigma(t) C)^{T}(I-P) \Sigma(t) C \sigma(t)\right] \\
& \leq \operatorname{trace}\left[C^{T} \sum(t)^{T} \Sigma(t) C \sigma(t)\right] \\
& =2 \sigma(t)^{2} \sum_{i=1}^{s} d_{i}\left(\kappa_{i}^{2}+\tau_{i}^{2}-\kappa_{i} \tau_{i}\right) \\
& \leq 2 s \sigma(t)^{2} d_{M}\left(\widehat{\kappa}^{2}+\widehat{\tau}^{2}-\check{\kappa} \check{\tau}\right), \\
& e(t)^{T} f\left(\varrho_{\imath}, e(t), e(t-a)\right)+\frac{1}{2}\left|g\left(\varrho_{\imath}, e(t), e(t-a)\right)\right|^{2} \\
& \leq e(t)^{T}[(I-P) \Pi(t) e(t)]+e(t)^{T}[(I-P) \Sigma(t) e(t-a)]+ \\
& \operatorname{trace}\left[((I-P) \Sigma(t) C)^{T}(I-P) \Sigma(t) C \sigma(t)\right] \\
& \leq f_{1}\left(\delta_{1}\right)+f_{2}\left(\delta_{2}\right)+f_{3}\left(\delta_{3}\right)+2 n \sigma(t)^{2} d_{M}\left(\widehat{\kappa}^{2}+\widehat{\tau}^{2}-\check{\kappa} \check{\tau}\right),
\end{aligned}
$$

and hence it gets the following result.

Theorem 1. If $\mathscr{G}\left(\varrho_{l}\right)$ is connected and if

$$
\begin{aligned}
& g_{1}(t)=\frac{\widehat{\tau} \lambda_{M}}{\widehat{\kappa} \lambda_{M}} g_{3}(t), \\
& g_{3}(t)=\frac{\widehat{\kappa}}{\widehat{\tau}}-\frac{\check{\kappa} \lambda_{2}}{\widehat{\tau} \lambda_{M}} h_{2}(t)+g_{2}(t), \\
& \sigma(t)^{2}<\frac{v+\check{\tau} \lambda_{2}-\widehat{\kappa} \lambda_{M}}{2 n d_{M}\left(\widehat{\kappa}^{2}+\widehat{\tau}^{2}-\check{\kappa} \check{\tau}\right)} e(t-a)^{T} e(t-a),
\end{aligned}
$$

where $\nu$ is a real number, and $\nu<1,2 q_{l} \sigma_{l} \leq 1$, then system (31) is asymptotically stable, where $\lambda_{2}$ is the second largest eigenvalue of $L, A-2=\operatorname{diag}\left\{\omega_{1}, \omega_{2}, \ldots, \omega_{m}\right\}-\Gamma$ is a nonsingular M-matrix, and $\left(q_{1}, q_{2}, \ldots, q_{m}\right)^{T}:=A^{-1} \overrightarrow{1}$. Therefore, system (31) is the BCT consensus.

Proof. Define the function $V: R^{n} \times R_{+} \times S \longrightarrow R_{+}$by

$$
V\left(\delta, \varrho_{\imath}, t\right)=q_{\imath} \sum_{j=1}^{3} V_{j}=q_{\imath} \sum_{j=1}^{3} \delta_{j}^{T} \delta_{j},
$$

with $c_{1}=\min _{\iota \in \mathscr{T}} q_{l}$ and $c_{2}=\max _{\iota \in \mathscr{T}} q_{l}$. Applying the generalised It $\hat{o}$ formula to $e^{\varsigma_{j} t} V_{j}$, it holds that

$$
\begin{aligned}
L V_{j} \leq & 2 q_{\iota}\left[\delta_{j}(t)^{T} f\left(\varrho_{\imath}, \delta_{j}(t), \delta_{j}(t-a)\right)\right. \\
& \left.+\frac{1}{2}\left|g\left(\varrho_{\imath}, \delta_{j}(t), \delta_{j}(t-a)\right)\right|^{2}\right]+\sum_{r=1}^{m} \gamma_{\iota r} q_{\iota} \delta_{j}(t)^{T} \delta_{j}(t),
\end{aligned}
$$

and hence

$$
\begin{aligned}
& L V \leq 2 q_{\iota}\left[e(t)^{T} f\left(\varrho_{\imath}, e(t), e(t-a)\right)+\frac{1}{2}\left|g\left(\varrho_{\imath}, e(t), e(t-a)\right)\right|^{2}\right] \\
& +\sum_{r=1}^{m} \gamma_{t r} q_{\imath} e(t)^{T} e(t) \\
& \leq 2 q_{\iota}\left[-\check{\kappa} \lambda_{2}+g_{i}(t) \hat{\kappa} \lambda_{M}\right] \delta_{1}(t)^{T} \delta_{1}(t)-\check{\kappa} \lambda_{2} \delta_{1}(t-a)^{T} \delta_{1}(t-a) \\
& +\sum_{r=1}^{m} \gamma_{t r} q_{l} \delta_{1}(t)^{T} \delta_{1}(t)+2 q_{\iota}\left[\widehat{\kappa} \lambda_{M}-h_{2}(t) \check{\kappa} \lambda_{2}-\check{\tau} \lambda_{2}\right. \\
& \left.+\widehat{\tau} \lambda_{M} g_{2}(t)\right] \delta_{2}(t)^{T} \delta_{2}(t) \\
& +\left(\widehat{\kappa} \lambda_{M}-\check{\tau} \lambda_{2}\right) \delta_{2}(t-a)^{T} \delta_{2}(t-a)+2 n \sigma(t)^{2} d_{M}\left(\widehat{\kappa}^{2}+\widehat{\tau}^{2}-\check{\kappa} \check{\tau}\right) \\
& +\sum_{r=1}^{m} \gamma_{l r} q_{\imath} \delta_{2}(t)^{T} \delta_{2}(t) \\
& {\left[-\check{\tau} \lambda_{2}+\widehat{\tau} \lambda_{M} g_{3}(t)\right] \delta_{3}(t)^{T} \delta_{3}(t)-\check{\tau} \lambda_{2} \delta_{3}(t-a)^{T} \delta_{3}(t-a)} \\
& +\sum_{r=1}^{m} \gamma_{l r} q_{l} \delta_{3}(t)^{T} \delta_{3}(t) \\
& \leq-\delta_{1}(t)^{T} \delta_{1}(t)-\check{\kappa} \lambda_{2} \delta_{1}(t-a)^{T} \delta_{1}(t-a)-\delta_{2}(t)^{T} \delta_{2}(t) \\
& +\left(\widehat{\kappa} \lambda_{M}-\check{\tau} \lambda_{2}\right) \delta_{2}(t-a)^{T} \delta_{2}(t-a)-\delta_{3}(t)^{T} \\
& \delta_{3}(t)-\check{\tau} \lambda_{2} \delta_{3}(t-a)^{T} \delta_{3}(t-a)+2 n \sigma(t)^{2} d_{M}\left(\widehat{\kappa}^{2}+\widehat{\tau}^{2}-\check{\kappa} \check{\tau}\right) \\
& \leq-e(t)^{T} e(t)+\left(\widehat{\kappa} \lambda_{M}-\tilde{\tau} \lambda_{2}\right) e(t-a)^{T} e(t-a) \\
& \leq-e(t)^{T} e(t)+\nu e(t-a)^{T} e(t-a),
\end{aligned}
$$

and according to Lemma 1, system (31) is asymptotically stable.

So, it holds that

$$
\lim _{t \rightarrow \infty} E\|e(t)\|=0,
$$

and that system (31) is the BCT consensus. 


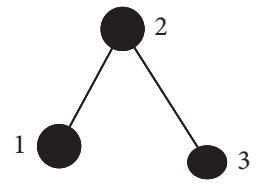

$Q_{1}$

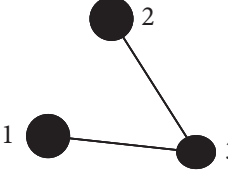

$Q_{2}$

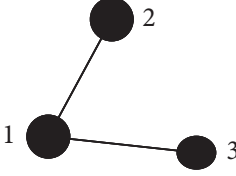

$Q_{3}$

Figure 1: The topologies of MAS (21).

TABLE 1: The values of curvature functions and torsion functions with different topologies.

\begin{tabular}{lccc}
\hline$\kappa$ and $\tau$ values of $\kappa$ and $\tau$ topologies & $\varrho_{1}$ & $\varrho_{2}$ & $\varrho_{3}$ \\
\hline$\left(\kappa_{1}, \tau_{1}\right)$ & $(0.1,0.21)$ & $(0.2,0.1)$ & $(0.25,0.23)$ \\
$\left(\kappa_{2}, \tau_{2}\right)$ & $(0.2,0.21)$ & $(0.33,0.22)$ & $(0.31,0.2)$ \\
$\left(\kappa_{3}, \tau_{3}\right)$ & $(0.23,0.2)$ & $(0.2,0.1)$ & $(0.25,0.21)$ \\
\hline
\end{tabular}
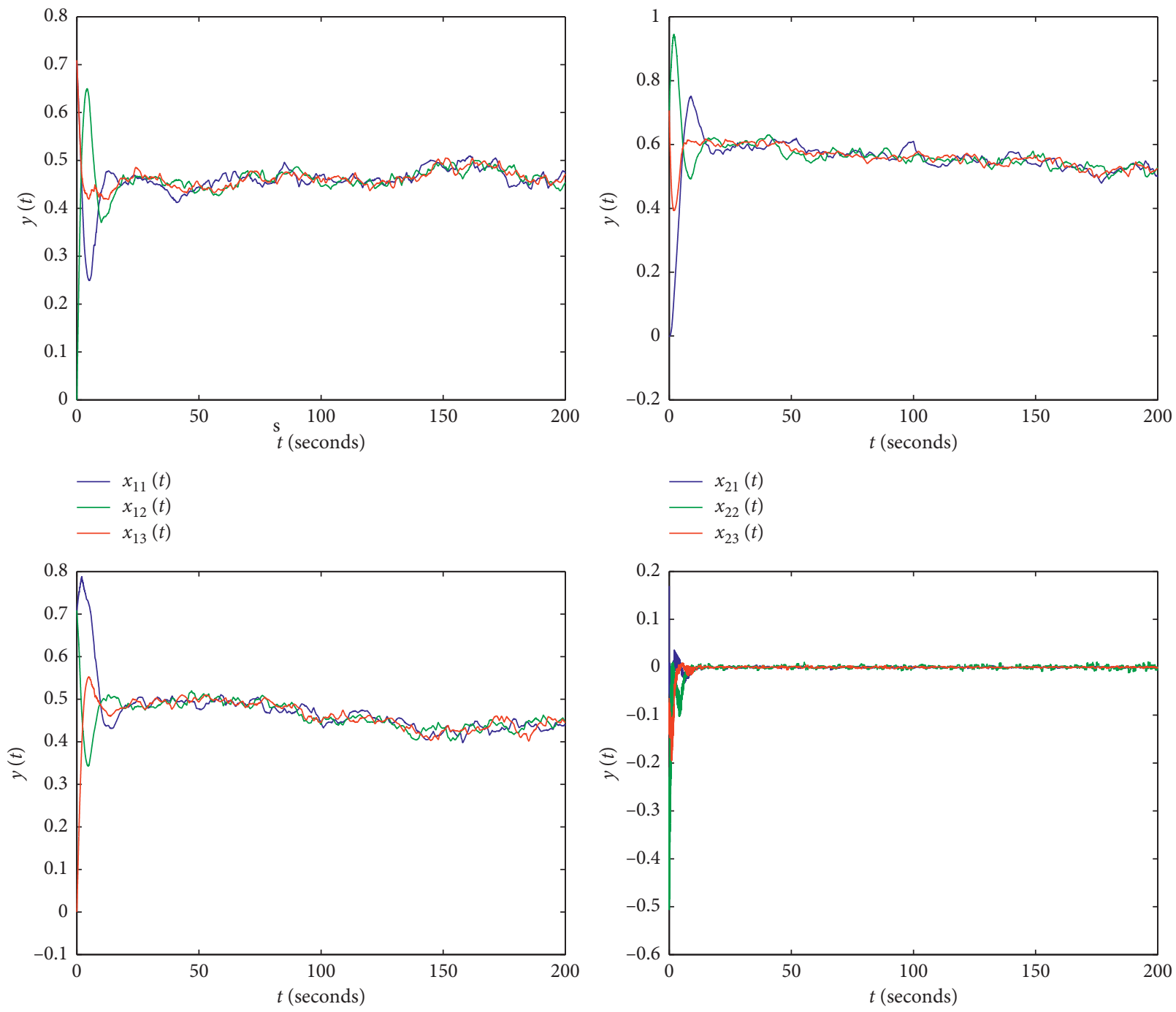

$-x_{31}(t)$
$-x_{32}(t)$
$-x_{33}(t)$

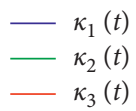

Figure 2: Component coordinates of vectors $\alpha_{1}, \alpha_{2}$, and $\alpha_{3}$ converging to a same value. 

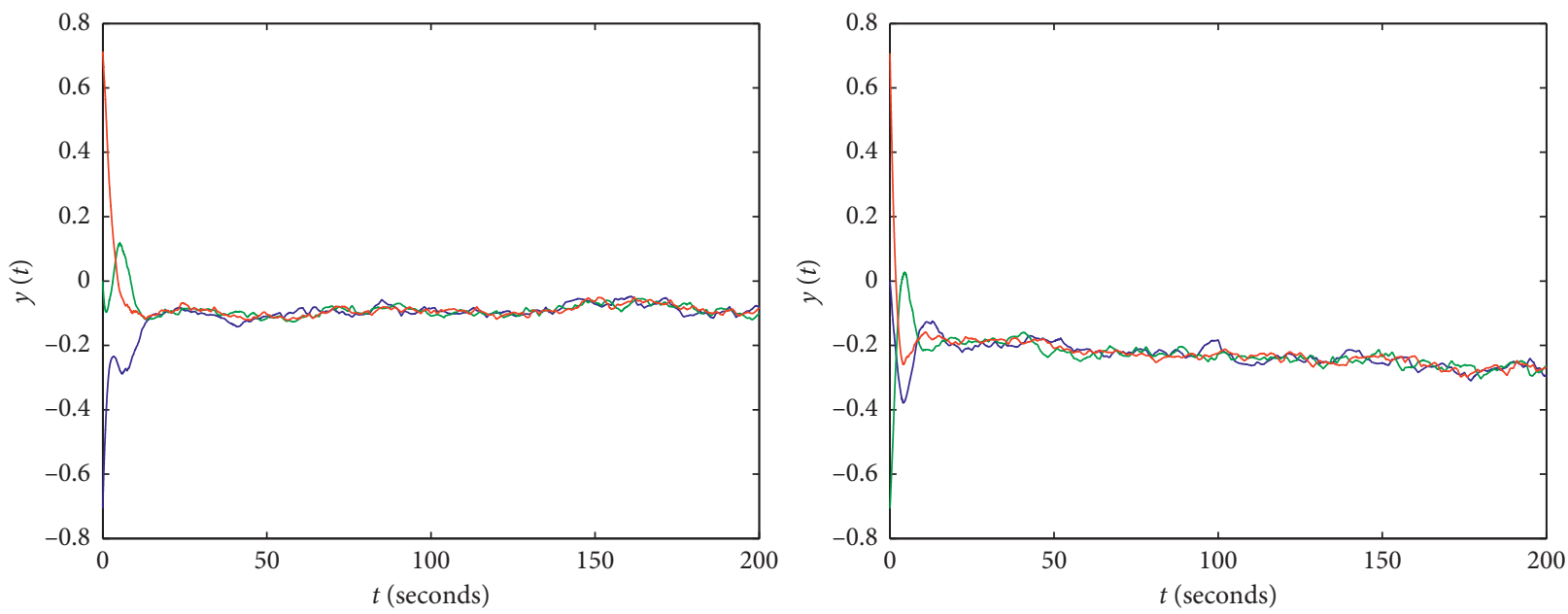

$\begin{array}{r}y_{11}(t) \\ -\quad y_{12}(t) \\ \hline\end{array}$

$y_{21}(t)$

- $y_{22}(t)$

$-y_{33}(t)$
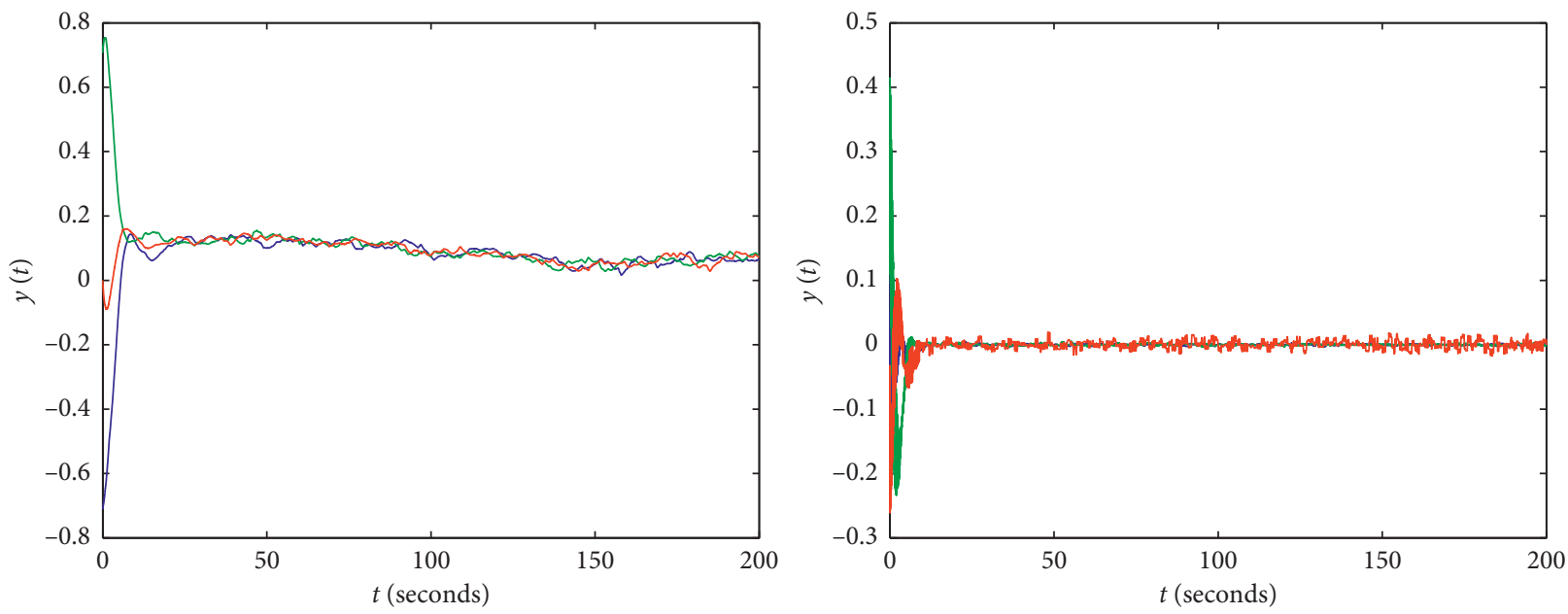

$-y_{31}(t)$
$-y_{32}(t)$
$-\mathrm{y}_{33}(t)$

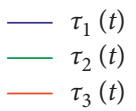

Figure 3: Component coordinates of vectors $\beta_{1}, \beta_{2}$, and $\beta_{3}$ converging to a same value.

Theorem 2. If $\mathscr{G}$ is connected and if

$$
\begin{aligned}
& g_{1}(t)=\frac{\widehat{\tau} \lambda_{M}}{\widehat{\kappa} \lambda_{M}} g_{3}(t), \\
& g_{3}(t)=\frac{\widehat{\kappa}}{\widehat{\tau}}-\frac{\check{\kappa} \lambda_{2}}{\widehat{\tau} \lambda_{M}} h_{2}(t)+g_{2}(t), \\
& \sigma(t)^{2}<\frac{v+\check{\tau} \lambda_{2}-\widehat{\kappa} \lambda_{M}}{2 n d_{M}\left(\widehat{\kappa}^{2}+\widehat{\tau}^{2}-\check{\kappa} \check{\tau}\right)} e(t-a)^{T} e(t-a),
\end{aligned}
$$

where $v$ is a real number, and $\nu<1, v e^{a c_{1}}=1$, then system (31) is asymptotically stable. Therefore, system (31) is the BCT consensus.
Proof. Since

$$
\begin{aligned}
\lambda e^{\varsigma_{j} t} V_{j}+e^{\varsigma_{j} t} L V_{j} \leq \varsigma_{j} e^{\varsigma_{j} t} V_{j}+e^{\varsigma_{j} t} & \\
\cdot & {\left[f_{j}(t)-\check{\kappa} \lambda_{2} \delta_{1}(t-a)^{T} \delta_{1}(t-a)+2 n \sigma(t)^{2} c_{3}\right], }
\end{aligned}
$$

where $c_{3}=d_{M}\left(\widehat{\kappa}^{2}+\widehat{\tau}^{2}-\check{\kappa} \check{\tau}\right)$, then

$$
\begin{aligned}
c_{1} e^{\varsigma_{j} t} E|e(t)|^{2} \leq & c_{2} E\left|\xi_{j}(0)\right|^{2} \\
& +E\left[\int_{0}^{t} e^{\varsigma_{j} s}\left(-e(s)^{T} e(s)+v e(s-a)^{T} e(s-a) \mathrm{d} s\right)\right],
\end{aligned}
$$

where 

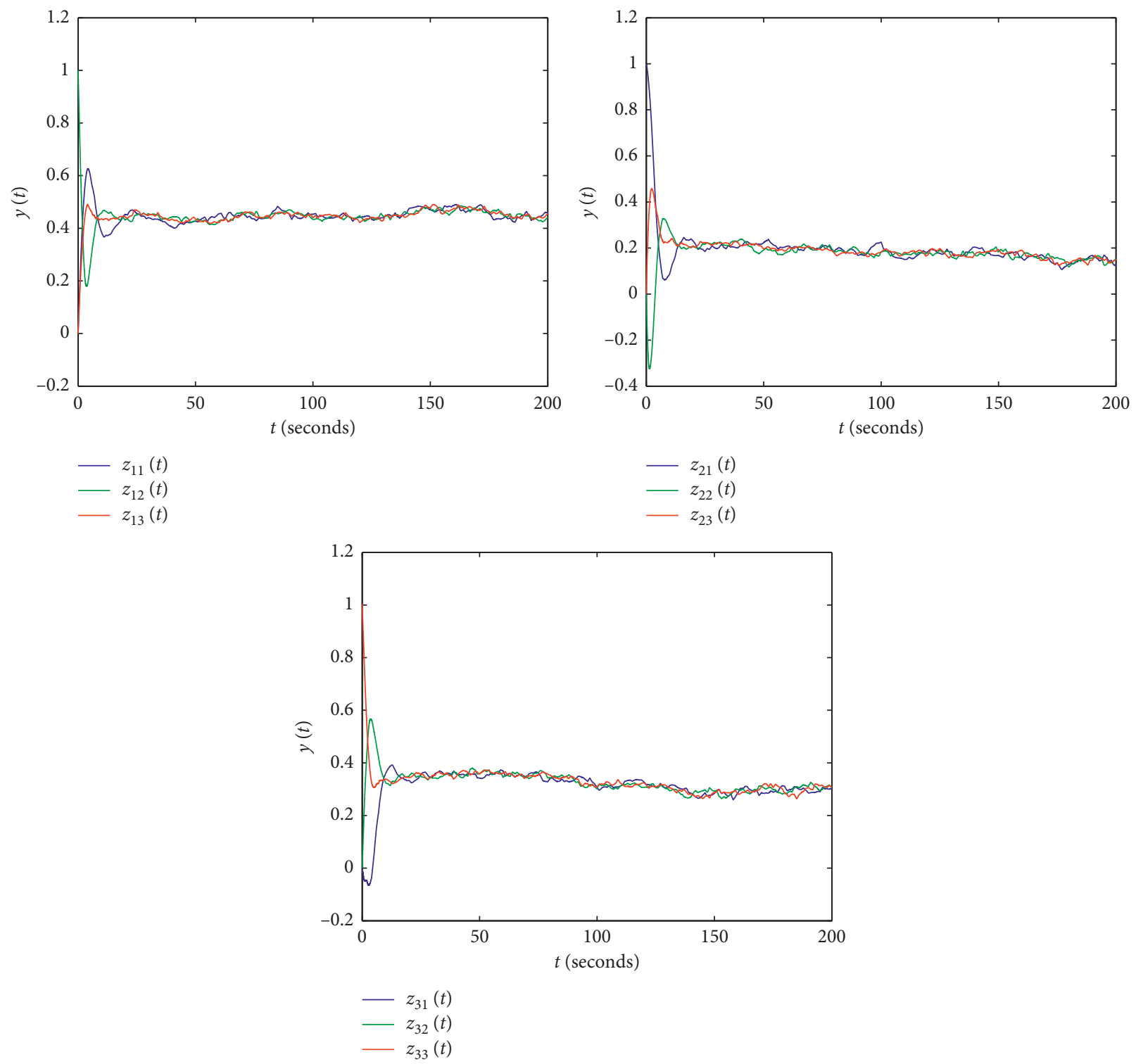

FIGURE 4: Component coordinates of vectors $\gamma_{1}, \gamma_{2}$, and $\gamma_{3}$ converging to a same value.

$$
\begin{aligned}
& \int_{0}^{t} e^{\varsigma_{1} s}\left(\left[v e(s-a)^{T} e(s-a)\right] \mathrm{d} s\right) \\
& \leq v e^{a \varsigma_{1}}\left[\int_{-a}^{t} e^{\varsigma_{1}(s-a)}\left(v e(s-a)^{T} e(s-a)\right) \mathrm{d}(s-a)\right] \\
& =v e^{a \varsigma_{1}} \int_{0}^{t} e^{\varsigma_{1} s}\left(e(s)^{T} e(s)\right) \mathrm{d} s+v e^{a \varsigma_{1}} \int_{-a}^{0} e^{\varsigma_{1} s}\left(e(s)^{T} e(s)\right) \mathrm{d} s .
\end{aligned}
$$

Since it holds that $v e^{a \varsigma_{1}}=1$,

$$
c_{1} e^{\varsigma_{j} t} E|e(t)|^{2} \leq c_{2} E\left|\xi_{j}(0)\right|^{2}+v e^{a \varsigma_{1}} E\left\|\xi_{1}\right\|^{2},
$$

and $\varsigma_{1}$ satisfies that

$$
-\frac{\lambda_{1}}{2} \check{\kappa}+\frac{1}{2} e^{a \varsigma_{1}}\left(\lambda_{M} k_{1} \widehat{\kappa}-\lambda_{1} \check{\kappa}\right)=0,
$$

and according to Lemma 2, system (31) is asymptotically stable.
So, it holds that

$$
\lim _{t \longrightarrow \infty} E\|e(t)\|=0
$$

and that system (31) is the BCT consensus.

\section{A Numerical Example}

To illustrate the effect of the methods in the main results, an example is proposed in the following.

Example 1. Consider a connected MAS (21) with three agents, and the topologies of (21) are shown in Figure 1, where $\mathscr{V}=\left\{v_{1}, v_{2}, v_{3}\right\}$. According to the different topologies, the related curvature functions and torsion functions are provided in Table 1. 


$$
\begin{aligned}
X(t) & =\left\{r_{1}(t), r_{2}(t), r_{3}(t)\right\}^{T}, \\
r_{1}(0) & =\{17,0,0\}^{T}, \\
r_{2}(0) & =\{50,0,0\}^{T}, \\
r_{3}(0) & =\{20,0,0\}^{T} .
\end{aligned}
$$

The related matrices of equation (21) are listed below.

$$
\begin{aligned}
& \Pi_{12}\left(\varrho_{1}\right)=\left[\begin{array}{ccc}
0.1 & -0.1 & 0 \\
-0.1 & 0.2 & -0.1 \\
0 & -0.25 & 0.25
\end{array}\right]=-\Pi_{21}\left(\varrho_{1}\right) \text {, } \\
& \Pi_{23}\left(\varrho_{1}\right)=\left[\begin{array}{ccc}
0.21 & -0.21 & 0 \\
-0.05 & 0.1 & -0.05 \\
0 & -0.22 & 0.22
\end{array}\right]=-\Pi_{32}\left(\varrho_{1}\right) \text {, } \\
& \Pi_{12}\left(\varrho_{2}\right)=\left[\begin{array}{ccc}
0.2 & 0 & -0.2 \\
0 & 0.33 & -0.33 \\
-0.31 & -0.31 & 0.62
\end{array}\right]=-\Pi_{21}\left(\varrho_{2}\right) \text {, } \\
& \Pi_{23}\left(\varrho_{2}\right)=\left[\begin{array}{ccc}
0.21 & 0 & -0.21 \\
0 & 0.22 & -0.22 \\
-0.11 & -0.11 & 0.22
\end{array}\right]=-\Pi_{32}\left(\varrho_{2}\right) \text {, } \\
& \Pi_{12}\left(\varrho_{3}\right)=\left[\begin{array}{ccc}
0.23 & -0.115 & -0.115 \\
-0.2 & 0.2 & 0 \\
-0.25 & 0 & 0.25
\end{array}\right]=-\Pi_{21}\left(\varrho_{3}\right) \text {, } \\
& \Pi_{23}\left(\varrho_{3}\right)=\left[\begin{array}{ccc}
0.2 & -0.1 & -0.1 \\
-0.1 & 0.1 & -0 \\
-0.21 & 0 & 0.21
\end{array}\right]=-\Pi_{32}\left(\varrho_{3}\right) \text {, } \\
& \Sigma_{1}\left(\varrho_{1}\right)=\left[\begin{array}{ccc}
0.1 & -0.1 & 0 \\
-0.2 & 0.4 & 0.2 \\
0 & -0.25 & 0.25
\end{array}\right] \text {, } \\
& \Sigma_{2}\left(\varrho_{1}\right)=\left[\begin{array}{ccc}
0.01 & -0.01 & 0 \\
0.1 & -0.2 & 0.1 \\
0 & 0.03 & -0.03
\end{array}\right] \text {, } \\
& \Sigma_{3}\left(\varrho_{1}\right)=\left[\begin{array}{ccc}
0.21 & -0.21 & 0 \\
-0.1 & 0.2 & -0.1 \\
0 & -0.22 & 0.22
\end{array}\right] \text {, } \\
& \Sigma_{1}\left(\varrho_{2}\right)=\left[\begin{array}{ccc}
0.2 & 0 & -0.2 \\
0 & 0.33 & -0.33 \\
-0.31 & -0.31 & 0.62
\end{array}\right] \text {, } \\
& \Sigma_{2}\left(\varrho_{2}\right)=\left[\begin{array}{ccc}
0.01 & 0 & -0.01 \\
0 & -0.11 & 0.11 \\
0.11 & 0.11 & -0.22
\end{array}\right] \text {, } \\
& \Sigma_{3}\left(\varrho_{2}\right)=\left[\begin{array}{ccc}
0.21 & 0 & -0.21 \\
0 & 0.22 & -0.22 \\
-0.2 & -0.2 & 0.4
\end{array}\right] \text {, } \\
& \Sigma_{1}\left(\varrho_{3}\right)=\left[\begin{array}{ccc}
0.46 & -0.23 & -0.23 \\
-0.2 & 0.2 & 0 \\
-0.25 & 0 & 0.25
\end{array}\right] \text {, } \\
& \Sigma_{2}\left(\varrho_{3}\right)=\left[\begin{array}{ccc}
-0.06 & 0.03 & 0.03 \\
0.1 & -0.1 & 0 \\
0.04 & 0 & -0.04
\end{array}\right] \text {, } \\
& \Sigma_{3}\left(\varrho_{3}\right)=\left[\begin{array}{ccc}
0.4 & -0.2 & -0.2 \\
-0.1 & 0.1 & 0 \\
-0.21 & 0 & 0.21
\end{array}\right] \text {. }
\end{aligned}
$$

From the following Laplacian matrices:

$$
\begin{aligned}
& L\left(\varrho_{1}\right)=\left[\begin{array}{ccc}
1 & -1 & 0 \\
-1 & 2 & -1 \\
0 & -1 & 1
\end{array}\right], \\
& L\left(\varrho_{2}\right)=\left[\begin{array}{ccc}
1 & 0 & -1 \\
0 & 1 & -1 \\
-1 & -1 & 2
\end{array}\right], \\
& L\left(\varrho_{3}\right)=\left[\begin{array}{ccc}
2 & -1 & -1 \\
-1 & 1 & 0 \\
-1 & 0 & 1
\end{array}\right],
\end{aligned}
$$

it obtains that $\lambda_{2}=1, \lambda_{M}=3$. Suppose that $v=0.8$, $h_{2}(t)=0.5$,

$$
\begin{aligned}
\sigma\left(t, \varrho_{1}\right)^{2}<0.2 e(t-a)^{T} e(t-a), \\
\sigma\left(t, \varrho_{2}\right)^{2}<\frac{1}{72} e(t-a)^{T} e(t-a), \\
\sigma\left(t, \varrho_{3}\right)^{2}<0.12 e(t-a)^{T} e(t-a), \\
g_{1}((t, \varrho))=\frac{1}{3}, \\
g_{2}((t, \varrho))=\frac{\widehat{\kappa}}{6 \widehat{\tau}} \\
g_{3}((t, \varrho))=\frac{\widehat{\kappa}}{\widehat{\tau}}, \\
\Gamma=\left[\begin{array}{ccc}
-2 & 1 & 1 \\
1 & -2 & 1 \\
1 & 1 & -2
\end{array}\right] .
\end{aligned}
$$

According to Theorem 1, under the control of protocol (17), MAS (21) achieves the BCT consensus (see the simulation results of Figures 2-4).

Figures 2-4 show that under protocol (18), every component coordinate of vectors $\alpha_{i}(t), \beta_{i}(t), \gamma_{i}(t)$ converges to a common value, respectively, curvature functions and torsion functions converge to a value, respectively, and all agents converge to the BCT consensus. In some applications, the protocol ensures that all agents keep the same trajectories; in the meanwhile, agents can also keep nonzero distances.

\section{Conclusion}

In this paper, we have studied the consensus problem in the sense of torsion and curvature for stochastic MAS with Markovian switching topology and time delay. A kind of distributed consensus protocol has been designed for MAS, and we have gotten the MAS which is updating upon torsion function and curvature function, where the protocol is based on the Frenet-Serret formulas. By employing the Lyapunov functional method and the stochastic approaching, several 
consensus criteria have been obtained, the results have shown that the protocol can ensure the system achieves the consensus, and the criteria have been analysed and illustrated by simulations. However, some challenging problems have not been considered in this paper, such as MAS in the small-world framework or systems with unknown partial Markovian transformation rate, and the related consensus problems will be studied by us in the future.

\section{Data Availability}

The data used to support the findings of this study are included within the article.

\section{Conflicts of Interest}

The authors declare that they have no conflicts of interest.

\section{Acknowledgments}

This study was partially supported by the National Natural Science Foundation of China (61673257), Fund of Zhejiang Provincial Education Department (Y201941722), Cultivation Fund of Taizhou University (2019PY07), Research Initiation Fund of Taizhou University (0104010004), and Fund of Zhejiang College Student of Science and Technology Innovation Activity Program and New Talent Program (2019R436008).

\section{References}

[1] X. Li and J. Wang, "Fault-tolerant tracking control for a class of nonlinear multi-agent systems," Systems \& Control Letters, vol. 135, p. 104576, 2020.

[2] G. Wen, X. Zhai, Z. Peng, and A. Rahmani, "Fault-tolerant secure consensus tracking of delayed nonlinear multi-agent systems with deception attacks and uncertain parameters via impulsive control," Communications in Nonlinear Science and Numerical Simulation, vol. 82, p. 105043, 2020.

[3] Z. Yu, S. Yu, and H. Jiang, "Consensus of multi-agent systems with finite-time and fixed-time observation," Information Sciences, vol. 512, pp. 909-928, 2020.

[4] Y. Chang, S. Zhang, N. D. Alotaibi, and A. F. Alkhateeb, "Observer-based adaptive finite-time tracking control for a class of switched nonlinear systems with unmodeled dynamics," IEEE Access, vol. 8, 2020.

[5] L. Ma, G. Zong, X. Zhao, and X. Huo, "Observed-based adaptive finite-time tracking control for a class of nonstrictfeedback nonlinear systems with input saturation," Journal of the Franklin Institute, vol. 357, no. 16, pp. 11518-11544, 2020.

[6] F. Chen and W. Ren, "On the control of multi-agent systems: a survey(Review)," Foundations and Trends in Systems and Control, vol. 6, pp. 1-164, 2019.

[7] D. Yu, L. Dong, and H. Yan, "Adaptive sliding mode control of multi-agent relay tracking systems with disturbances," Journal of Control and Decision, 2019.

[8] J. Zhang, S. Chai, B. Zhang, and G. Liu, "Relay cooperative tracking control of networked nonlinear multi-agent systems with communication delays: a data-driven method," Neurocomputing, vol. 363, pp. 9-16, 2019.

[9] R. Rădulescu, P. Mannion, D. M. Roijers, and A. Nowé, "Multi-objective multi-agent decision making: a utility-based analysis and survey," Autonomous Agents and Multi-Agent Systems, vol. 34, pp. 1-52, 2020.

[10] Y. Xu, X. Wu, N. Li, L. Liu, C. Xie, and C. Li, "Fixed-time synchronization of complex networks with a simpler nonchattering controller," IEEE Transactions on Circuits and Systems II: Express Briefs, vol. 67, no. 4, pp. 700-704, 2020.

[11] Y. Zheng, J. Ma, and L. Wang, "Consensus of hybrid multiagent systems," IEEE Transactions on Neural Networks and Learning Systems, vol. 29, no. 4, pp. 1359-1365, 2018.

[12] M. He, J. Mu, and X. Mu, "Ho leader-following consensus of nonlinear multi-agent systems under semi-Markovian switching topologies with partially unknown transition rates," Information Sciences, vol. 513, pp. 168-179, 2020.

[13] M. Li, F. Deng, and H. Ren, "Scaled consensus of multi-agent systems with switching topologies and communication noises," Nonlinear Analysis: Hybrid Systems, vol. 36, p. 100839, 2020.

[14] J. Wang, G. Wen, Z. Duan, and J. L. Jü, "Stochastic consensus control integrated with performance improvement: a consensus region-based approach," IEEE Transactions on Industrial Electronics, vol. 67, no. 4, pp. 3000-3012, 2020.

[15] H. Lu, J. Zhang, L. Jing, and W. Ying, "Neural-network-based adaptive quasi-consensus of nonlinear multi-agent systems with communication constrains and switching topologies," Nonlinear Analysis: Hybrid Systems, vol. 35, p. 100833, 2020.

[16] S. Luo, F. Deng, and X. Zhao, "Analysis of linear asynchronous hybrid stochastic systems and its application to multi-agent systems with Markovian switching topologies," International Journal of Systems Science, vol. 50, no. 9, pp. 1757-1770, 2019.

[17] X. Li, C. Zhou, J. Zhou, Z. Wang, and J. Xia, "Couple-group L2-Lo consensus of nonlinear multi-agent systems with Markovian switching Topologies $L_{2}-L_{\infty}$ Consensus of nonlinear multi-agent systems with Markovian switching topologies," International Journal of Control, Automation and Systems, vol. 17, no. 3, pp. 575-585, 2019.

[18] M. Fang, C. Zhou, X. Huang, X. Li, and J. Zhou, " $H_{\infty}$ couplegroup consensus of stochastic multi-agent systems with fixed and Markovian switching communication topologies," Chinese Physics B, vol. 28, 2019.

[19] D. Tong, C. Xu, Q. Chen, W. Zhou, and Y. Xu, "Sliding mode control for nonlinear stochastic systems with Markovian jumping parameters and mode-dependent time-varying delays," Nonlinear Dynamics, vol. 100, no. 2, pp. 1343-1358, 2020.

[20] D. Tong, Q. Chen, W. Zhou, and Y. Xu, "Adaptive state estimation of Markov switched neural networks driven by Lévy noise evy noise," Transactions of the Institute of Measurement and Control, vol. 42, no. 2, pp. 330-336, 2020.

[21] A. Parivallal, R. Sakthivel, R. Amsaveni, F. Alzahrani, A. S. Alshomrani, and S. Ali, "Observer-based memory consensus for nonlinear multi-agent systems with output quantization and Markov switching topologies," Physica A: Statistical Mechanics and Its Applications, vol. 551, p. 123949, 2020.

[22] R. Sakthivel, A. Parivallal, B. Kaviarasan, H. Lee, and Y. Lim, "Finite-time consensus of Markov jumping multi-agent systems with time-varying actuator faults and input saturation," ISA Transactions, vol. 83, pp. 89-99, 2018.

[23] J.-S. Song and X.-H. Chang, "Ho controller design of networked control systems with a new quantization structure cocontroller design of networked control systems with a new quantization structure," Applied Mathematics and Computation, vol. 376, p. 125070, 2020. 
[24] D. Xie, L. Shi, and F. Jiang, "Second-order group consensus for linear multi-agent systems with average dwell time switching," Transactions of the Institute of Measurement and Control, vol. 41, no. 2, pp. 484-493, 2019.

[25] J. Gao, J. Li, H. Pan, Z. Wu, X. Yin, and H. Wang, "Consensus via event-triggered strategy of nonlinear multi-agent systems with markovian switching topologies," ISA Transaction, vol. 104, pp. 122-129, 2019.

[26] J. Liu, Y. Zhang, Y. Yu, and C. Sun, "Fixed-time leader-follower consensus of networked nonlinear systems via event/self-triggered control," IEEE Transactions on Neural Networks and Learning Systems, vol. 31, pp. 5029-5037, 2020.

[27] Y. Xu, X. Wu, B. Mao, and J. Lü, "Fixed-time synchronization in the pth moment for time-varying delay stochastic multilayer networks," IEEE Transactions on Systems, Man, and Cybernetics: Systems, vol. 99, pp. 1-10, 2020.

[28] Y. Xu, X. Wu, B. Mao, and C. Xie, "A unified finite-/fixed-time synchronization approach to multi-layer networks," IEEE Transactions on Circuits and Systems II: Express Briefs, vol. 68, no. 1, pp. 311-315, 2021.

[29] X. Li and J. Wang, "Fault-tolerant tracking control for a class of nonlinear multi-agent systems," Systems \& Control Letters, vol. 135, 2020.

[30] A. Tariverdi, H. A. Talebi, and M. Shafiee, "Fault-tolerant consensus of nonlinear multi-agent systems with directed link failures, communication noise and actuator faults," International Journal of Control, vol. 94, no. 1, 2019.

[31] J. Mou, "Consensus of multiagent system in the sense of curvature and torsion," Mathematical Problems in Engineering, vol. 2019, Article ID 8084209, 11 pages, 2019.

[32] R. Zhou and J. Li, "Stochastic consensus of double-integrator leader-following multi-agent systems with measurement noises and time delays," International Journal of Systems Science, vol. 50, no. 2, pp. 365-378, 2019.

[33] X. Mao and C. Yuan, Stochastic Differential Equations with Markovian Switching, Imperial College Press, London, UK, 2006. 